\title{
Geographical Data Collection in Sensor Networks with Self-Organizing Transaction Cluster-Heads
}

\author{
Neeraj Rajgure ${ }^{1,2}$, Eric Platon ${ }^{1}$, Cristian Borcea ${ }^{2}$, Shinichi Honiden ${ }^{1}$ \\ ${ }^{1}$ National Institute of Informatics, 2-1-2 Hitotsubashi, 101-8430 Tokyo, Japan \\ ${ }^{2} \mathrm{New}$ Jersey Institute of Technology, University Heights, Newark, NJ 07102, USA \\ \{nmr2, borcea\}@njit.edu, \{platon, honiden\}@nii.ac.jp
}

\begin{abstract}
This paper proposes $2 \mathrm{G}$, a flexible and energy-efficient data collection protocol for sensor networks for increasing network lifetime. To this end, it integrates self-organizing data aggregation mechanisms based on geographical and clusterbased routing, and transaction cluster-head (TCH). A TCH is a location-based role, dynamically assigned to a node for the duration of handling a request-response transaction that targets its region of the network. TCH nodes collect raw sensor readings from their local regions and forward the answers containing aggregated data using geographical routing. A prototype of $2 \mathrm{G}$ was implemented on MICAz motes, and experimental results in realistic conditions proved that data collection reaches significantly higher delivery rates than with GEAR, the geographical routing protocol leveraged by $2 \mathrm{G}$. Additionally, simulation results for larger scale networks demonstrate that $2 \mathrm{G}$ outperforms GEAR in terms of network lifetime.
\end{abstract}

\section{Categories and Subject Descriptors}

C.2 [Computer-Communication Network]: Routing protocols; C.2.4 [Distributed Systems]: Distributed Applications

\section{General Terms \\ Design, Experimentation, Performance}

\section{Keywords}

Transaction cluster-heads, wireless sensor networks, self-organization, routing protocol

\section{INTRODUCTION}

Wireless sensor networks (WSN) aim at collecting information from our environment in a distributed manner and feed a computer-based system with raw or pre-processed sensed data. The efficient collection of distributed data

Permission to make digital or hard copies of all or part of this work for personal or classroom use is granted without fee provided that copies are not made or distributed for profit or commercial advantage and that copies bear this notice and the full citation on the first page. To copy otherwise, to republish, to post on servers or to redistribute to lists, requires prior specific permission and/or a fee.

SAC'09 March 8-12, 2009, Honolulu, Hawaii, U.S.A.

Copyright 2009 ACM 978-1-60558-166-8/09/03 ...\$5.00. depends both on data aggregation mechanisms and on the routing protocols, which have been active research areas for the past decade. Many routing protocols have been proposed during this period, such as low-power, self-adaptive, secure, or cluster-based routing $[6,13,1,7,10]$. However, not many protocols targeted the key issues of geographic routing and region-based data collection in WSN, which are based on the intrinsic spatial nature of these networks. Unlike geographical routing in ad hoc networks which forwards information toward a node specified by its coordinates, geographical protocols in WSN need to route requests toward a specific region of the environment to collect and potentially aggregate sensory information, and subsequently forward the answer to a base station. The GEAR (Geographical and Energy Aware Routing) protocol is a notable example of tackling geographical routing in WSN [15].

However, GEAR and similar protocols targeted node-tonode geographical routing, which is appropriate for typical ad hoc network scenarios. Therefore, their performance and flexibility in WSN were limited because they did not take advantage of data fusion schemes and cluster-based mechanisms to speed up data collection and reduce energy consumption. In addition, these mechanisms could be enhanced with self-organizing techniques to address flexibility issues in geographical data collection and routing.

This paper proposes $2 \mathrm{G}$ (second-generation GEAR), a region-based data collection protocol for WSN that augments GEAR with a self-organizing mechanism which dynamically assigns transaction cluster-heads (TCH) per request and per region. In response to a request, a TCH performs region-based aggregation and uses geographical routing to deliver the response. We implemented $2 \mathrm{G}$ on the MI$\mathrm{CAz}$ platform from Crossbow. Our field experiments with this prototype as well as simulation results using TOSSIM [8] demonstrated significant performance improvements compared to a basic approach in which data is collected from individual nodes using GEAR.

The rest of the paper is organized as follows. Section 2 overviews the GEAR routing protocol. In Section 3, we briefly discuss existing cluster-based mechanisms in WSN and the concept of role assignment. Sectiontransactioncluster describes the design of $2 \mathrm{G}$, with a special focus on using 'transaction cluster-heads' as a self-organizing mechanism to improve the performance of data collection. Experimental and simulation results are presented in Section 5. The paper concludes in Section 6 with a summary of contributions and a discussion of future work. 


\section{GEAR OVERVIEW}

GEAR is one of the representative geographical routing protocols in WSN [15]. It features self-adaptive routing based on geographical and local energy information. GEAR routes packets toward a target region trying to minimize the energy cost for each node along the route. GEAR differs from other energy-aware protocols with geographic routing such as the GAF module for ad hoc networks [14], as it exploits the abstraction of regions specific to WSN. GEAR also differs from protocols such as the power-aware zonebased routing, which requires knowledge about all nodes and some of their conditions [2]. GEAR only requires nodes to maintain knowledge about their in-range neighbors and the coarse-grained knowledge of regions for addressing (typically a list of region identifiers).

A request is sent to a region in the network from a base station (BS), which is assumed fixed at least for the duration of a request-response transaction. The BS can move between transactions without infringing the protocol (e.g., the BS could be a laptop). During the forwarding process, each node knows its region and chooses one of its neighbors as the next hop according to two conditions. First, the request must get closer to the target region (pure geographic routing). And second, the energy usage must be minimal (energy awareness). GEAR nodes maintain a small regionbased routing table, which is used only to determine how to get closer to the target region. Also, they update the table by dynamically learning the cost of reaching a target depending on the selected neighbor.

Once the request reaches a node of the target region, the node initiates a recursive routing mechanism. It consists of splitting the target region in four quarters and flooding them with the request. This approach is named 'restricted flooding' and aims at speeding up the dispatch of the request into the region. Each quarter can further be split with the same scheme in case of large and dense networks. GEAR features additional mechanisms that deal with particular cases, notably sparse networks (flooding) and no convergence due to energy wells (an approach based on changing to pure geographic routing). These specific mechanisms are not presented here as they are not essential to our paper. Once the request reaches the nodes of the target region, each node responds individually to the BS using the same algorithm.

Geographical routing protocols such as GEAR require a localization mechanism in order to determine the position of the nodes $[12,4,3]$. However, localization is a costly mechanism (e.g., several approaches require at least one flooding), but it is executed only once and then maintained reactively. The localization problem is out of the scope of this paper, but it remains a strong and necessary assumption.

GEAR relies on a setup protocol to initialize the knowledge of each node about the network and direct neighbors. A refresh protocol, very similar to the initial setup, runs periodically. The self-adaptation of routing depends on this protocol, and the autonomic update of routes is proportional to the path length: updating a path of $n$ hops requires the transmission of at least $n$ packets. This is an expensive operation and together with the lack of an aggregation scheme (i.e., each node-sensed data item is individually sent back to the BS) leads to an important decrease in performance. To improve on this situation, we propose to integrate a selfadaptive cluster-based mechanism in GEAR, where a region cluster-head is elected in response to a request in order to perform aggregation and then geographically forward the answer back to the BS.

\section{TRADITIONAL CLUSTER-BASED ROUT- ING IN WSN}

WSN target large-scale networks of thousands nodes. Data collection and routing on a per-node basis can be inefficient due to the amount of raw data generated by each node and the necessity to store too detailed routing information at nodes. Several approaches have been proposed to group nodes into clusters. Each cluster aggregates the data of its nodes (e.g., average) and sends the aggregate to the requester, thus significantly reducing the network load.

One important concept in cluster-based mechanisms is the role of a node [11]. Roles allow to distinguish among nodes and specialize their behavior in the routing protocol. Nodes in clusters are usually defined as cluster-heads $(\mathrm{CH})$ and regular cluster members $(\mathrm{CM})$. The $\mathrm{CH}$ receives requests for data and forwards them inside the cluster. Then, it collects replies, computes an aggregate response, and sends this response to the requester. Only $\mathrm{CHs}$ participate in the inter-cluster routing. CMs just send their sensor readings in reply to requests and forward packets from other members in case of multi-hop clusters. Members can also relay inter-cluster messages when two $\mathrm{CHs}$ cannot communicate directly. Typical cluster-based mechanisms are further described in related literature [5].

Common problems of these approaches are the lack of flexibility and the heavy tasks on the $\mathrm{CHs}$ that deplete their batteries quickly. The $\mathrm{CH}$ must handle all requests that arrive to the cluster. To solve them, existing solutions propose to change the roles of the nodes over time. Since CHs deplete their batteries faster than $\mathrm{CMs}$, a periodic $\mathrm{CH}$-election scheme is required. For example, the node with the lowest identifier and highest battery level defaults to the $\mathrm{CH}$. It should be noted, however, that this example suffers from security issues due to the ease of identity spoofing in WSN. Even worse, the election schemes are often based on costly negotiations that require extra communication. In addition, the self-organization of $\mathrm{CH}$ is not flexible enough, as the $\mathrm{CH}$ is chosen without considering its tasks and communication costs. For example, the election of a $\mathrm{CH}$ in a 'far corner' of a cluster can be inefficient as CMs must relay their messages to it, whereas it would have been faster and more economical to send data directly to the requester.

\section{2G DESIGN}

$2 \mathrm{G}$ leverages GEAR and cluster-based routing to provide a novel data collection protocol. A new role is introduced for nodes in $2 \mathrm{G}$, namely the transaction cluster-head (TCH). This role addresses the issues of flexibility and increased self-organizing capabilities. The role of $\mathrm{TCH}$ is to manage a request as a transaction. This role exists only for the duration of the transaction. The specifications of the TCH role are as follows:

Birth Once a request message reaches its target region, the first node that receives it becomes the $\mathrm{TCH}$ for this transaction (with simple exclusions in case two $\mathrm{TCH}$ arise, e.g. 'smaller' authorized network address).

Setup The TCH marks the data packet, such that nodes in the region know the TCH identity for this transaction 
and can sort out different transactions. The TCH then relays the request in the region by the original recursive routing of GEAR.

Collection The TCH waits for the replies from the region for a predefined amount of time. Once the time has elapsed, it applies application-specific aggregation functions on the received data.

Reply The TCH sends the aggregated data to the requester, using GEAR.

Death The $\mathrm{TCH}$ role is then dropped as the transaction is terminated.

An important property of the TCH role is that several nodes in the region can play this role at any time for handling concurrent transactions. This approach allows nodes to balance the extra load of playing a 'cluster-head'-like role, as our experimental results show in the next section.

The introduction of the TCH role in $2 \mathrm{G}$ implies the following features of the protocol.

- Geographical regions naturally become clusters that produce aggregated results.

- Requests from the base station and replies from regions (via TCH) follow the standard GEAR protocol.

- Different nodes become TCH for different transactions in a region, leading to a more uniform distribution of the energy consumption among nodes in that region. Since the TCH role depends on the actual energyaware route in GEAR, $2 \mathrm{G}$ guarantees this property (i.e., the same node does not end-up as TCH for every request coming from the $\mathrm{BS}$ ).

- The TCH self-election does not require any overhead communication. Therefore, $2 \mathrm{G}$ could significantly improve the network lifetime.

The model had initially included $\mathrm{CHs}$ in each region of the network (independent of the requests target regions) to leverage the properties of hierarchical routing [1]. We have eventually removed this mechanism as the problem of 'far corner', mentioned in the previous section, reduces the performance of GEAR between CHs.

Besides its benefits, $2 \mathrm{G}$ has also a drawback compared to GEAR. If a TCH dies during a transaction, no response is sent back and the energy associated with this transaction is wasted. As future work, we plan to add a corrective mechanism to cope with this issue.

\section{EXPERIMENTAL EVALUATION}

We have evaluated $2 \mathrm{G}$ using both a simulator and a prototype implemented on top of MICAz. The simulation aims at understanding the protocol behavior in large-scale networks, while the evaluation over a real platform aims at validating the protocol under realistic conditions in a field experiment.

\subsection{Evaluation settings and metrics}

The protocol has been developed for the TinyOS 2.0.2 operating system. It exists in two versions, namely plain GEAR and 2G. Plain GEAR is an implementation of the original protocol on real hardware. The experiments compared the performance of $2 \mathrm{G}$ and GEAR in terms of roundtrip time for requests to the network, network lifetime, and success rate of packet transmission. Table 1 compiles the main configuration parameters of our experiments.

Table 1: Settings

\begin{tabular}{|c|c|c|}
\hline & Simulation & On Motes \\
\hline Platform & TOSSIM & MICAz \\
\hline Nodes & 625 & 16 \\
\hline Network topology & \multicolumn{2}{|c|}{ Regular grid } \\
\hline $\begin{array}{c}\text { Source node } \\
\text { position }\end{array}$ & \multicolumn{2}{|c|}{ Bottom left node } \\
\hline $\begin{array}{c}\text { Target region } \\
\text { position }\end{array}$ & $\begin{array}{c}\text { Central node }+ \\
8 \text { neighbors }\end{array}$ & $\begin{array}{c}\text { Top right } \\
\text { square }\end{array}$ \\
\hline Target region & 9 nodes & 4 nodes \\
\hline Request period (s) & 24 & 4 \\
\hline Battery model & Linear and symmetric \\
\hline Init. battery level & Normalized to 250 units \\
\hline Low-batt. threshold & \multicolumn{2}{|c|}{50 units } \\
\hline Energy alert thresh. & \multicolumn{2}{|c|}{25 units } \\
\hline
\end{tabular}

The simulations of the two protocols were run on networks of size $100(10 \times 10)$ and 625 nodes $(25 \times 25)$ with periodic requests. Since the results are similar, we only report the figures for the 625-node network. All topologies are regular grids. This is a common choice of deployments in practice, and it also simplifies the analysis of the results. Cells of the grid for the hardware-based experiments measured 15 $\mathrm{cm}$ on the edge. The nodes were configured to accept communications only from their direct neighbors to reproduce a typical deployment, where radio coverage is an important economical factor. The battery model is a simple linear and symmetric decay function that estimates battery levels as follows: each time a node sends or receives a message, it decrements its battery level by one unit. Nodes inform the neighborhood about a low-battery level when 50 energy units remain out of 250 . When the energy is as low as 25 units, nodes inform the neighborhood that they should not be relied on anymore (subsequently, they are used only when there is no other alternative on the routing path).

The experiments provide an ad hoc and simple localization mechanism to provide nodes with position information. The mechanism relies on a discovery protocol that runs once at the setup of the network and a maintenance protocol that reacts to changes (e.g., dead nodes) to update the neighbors of the nodes in the network. The setup cost is not included in the evaluation. The maintenance cost, however, is part of the routing protocol, and consequently included in the evaluation metrics.

\subsection{Simulations}

Table 2 compiles the average values of the protocol metrics over 100 runs. The Round-Trip Time (RTT) indicates the time it takes for the BS to receive a response to a request. The values are similar, which means $2 \mathrm{G}$ does not introduce any overhead in terms of time performance. This result was expected due to the resemblance of the interaction patterns. The BS in Plain GEAR sends a request to a region and all members reply: the aggregation is done at the $\mathrm{BS}$ itself. When the BS in $2 \mathrm{G}$ sends a request to a region, all members reply to the TCH: the aggregation occurs at the TCH. Despite the difference on the aggregation point, all messages back to BS follow energy-efficient routes that are 
Table 2: Simulation results (625 nodes)

\begin{tabular}{|c|c|c|}
\hline & Plain GEAR & $2 \mathrm{G}$ \\
\hline Round-trip time (RTT) (ms) & 227.64 & 226.31 \\
\hline Rate of queries delivered to the target region & $84.70 \%$ & $89.28 \%$ \\
\hline Rate of responses back to the base station & $60.15 \%$ & $85.12 \%$ \\
\hline Accuracy of the collected value & $98.73 \%$ & $99.44 \%$ \\
\hline Average hop count & 11.29 & 2.65 \\
\hline Network lifetime (min) & 18.07 & 63.18 \\
\hline
\end{tabular}

similar between BS and TCH, thus leading to similar RTT.

The rate of requests delivered to the target region measures the effectiveness of delivery during the network lifetime. 2G performs better because its in-network aggregation saves battery power across the network and reduces the amount of data passing, consequently improving the overall routing performance. Furthermore, it leads to less network congestion. The rate of responses back to the base station is defined per successful request. The results show that $2 \mathrm{G}$ achieves significantly higher performance $(85.12 \%)$ compared to the plain GEAR (just $60.15 \%$ ). The explanation is similar to the one for the rate of requests, but in this case reducing the number of messages sent through the network has a much higher and direct impact on performance.

In addition to a higher success rate, it is notable that $2 \mathrm{G}$ allowed to increase significantly the network lifetime, from 18.07 min with plain GEAR to 63.18 min with $2 \mathrm{G}$ (3.5 times longer life). The accuracies of the collected data are similar due to the same aggregation function (average). These results demonstrate the benefits of using TCHs in $2 \mathrm{G}$, while not wasting bandwidth or energy to elect them.

The average hop count indicates the typical length of the route. In plain GEAR, each node of the target region replies to the BS, so the average is the sum of all route lengths divided by the number of nodes in the target region. In $2 \mathrm{G}$, only $\mathrm{TCH}$ replies to the BS, while other nodes need only reach $\mathrm{TCH}$. The average is thus the sum of the route length from $\mathrm{TCH}$ to $\mathrm{BS}$ added to the lengths from other nodes to $\mathrm{TCH}$, divided by the number of nodes in the target region. Based on this metrics, the results show that the TCH approach reduces significantly the average length of communication paths in the network from 11.29 to 2.65 , thus leading to significant energy savings on average. This is notably due to the self-organizing nature of TCHs, as any node in the target region is appropriate for the role, without constraining packets to converge toward a pre-defined cluster-head that usual cluster-based protocols would define. TCH appear at meaningful places with regards to the actual state of the network when the transaction starts.

Figures 1 and 2 show the evolution of the average number of hops to transmit a request and receive a response over time. As expected, the paths become longer over the network lifetime. As the energy at nodes on the shortest paths is consumed, the protocols adapt the route to exploit other nodes on alternative but longer paths. We also observe that TCHs in $2 \mathrm{G}$ lead to reduced communication costs, as the number of hops is significantly smaller than plain GEAR.

Figure 3 shows the distribution of the TCH roles in the target region over the lifetime of the network. Nodes are numbered from 1 to 9 , starting from the bottom-left of the region, toward the top-right. The BS is closest to node 1 , as

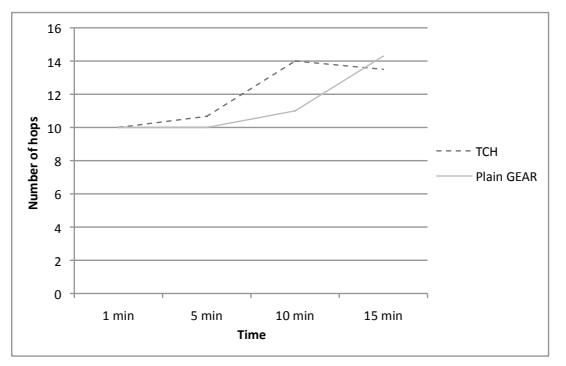

Figure 1: Average hops to transmit a request

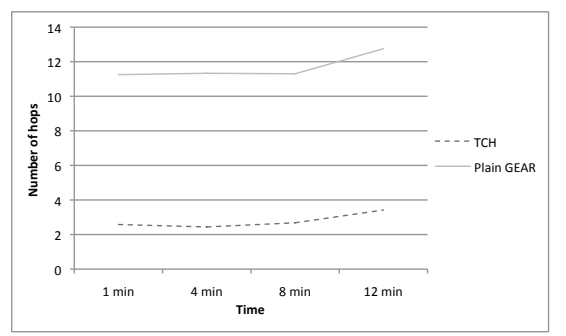

Figure 2: Average hops to transmit a response

the BS is in the bottom-left corner of the network. We observe that nodes on the border of the target region play more often the role of TCH (notably nodes 1, 2, and 7), whereas nodes inside the region almost never play this role, which is consistent with the TCH model. The graph shows that the only non-border node (number 5) does play the TCH role at some point of the simulation. As nodes are configured to process only messages from their direct neighbors, it means that node 5 becomes TCH only when surrounding nodes are either depleted or not on an energy-efficient route (note that nodes can range over about $100 \mathrm{~m}$ in free spaces). The self-organizing nature of TCH leverages the adaptive routing feature of the protocol to balance the overhead cost of serving as TCH for a given transaction.

\subsection{Field experiments}

We have executed $2 \mathrm{G}$ on the MICAz platform from Crossbow, which also runs TinyOS. These experiments used the same battery depletion model as the simulation, as the battery readings using the VoltageC API in TinyOS 2.0.2 were inconsistent. The limitation of this choice is that the network lifetime value cannot be measured accurately. Nevertheless, the experiments allowed to validate other metrics. Table 3 compiles the average results over 3 runs. 


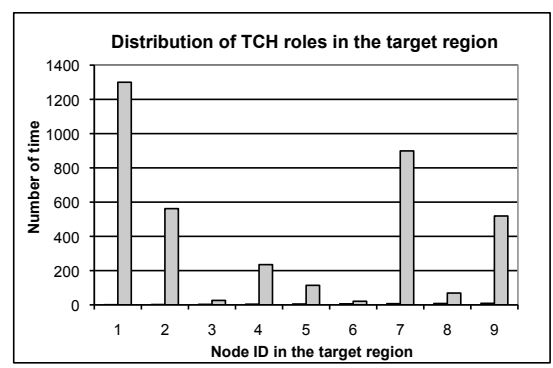

Figure 3: TCH role distribution

Table 3: Field experiments results (2G)

\begin{tabular}{|c|c|}
\hline Rate of queries to the target region & $91.33 \%$ \\
\hline Rate of responses to the base station & $93.11 \%$ \\
\hline Accuracy of the collected value & $99.75 \%$ \\
\hline Average hop count & 2.79 \\
\hline Network lifetime (min) & N/A (3.29) \\
\hline
\end{tabular}

These values validate the performance properties observed after simulations. The $2 \mathrm{G}$ prototype obtained higher success rates and accuracy for requests and responses compared to all simulations. There are two reasons for such good results: (1) the grid deployment provided reliable radio conditions, and (2) the size of the network was much smaller.

The measured network lifetime was only $3.29 \mathrm{~min}$, but this value relies on the simple battery depletion model, which does not take into account the real remaining energy. We report this value as it indicates that the 250 units of energy are spent on using the radio (on MICAz, sending and receiving have similar costs in our settings of non-attenuated signals [9]), and it demonstrates the self-organizing capabilities of the protocol, as shown in figure 3 .

\section{CONCLUSIONS}

The main contribution of this paper is the design, implementation, and evaluation of $2 \mathrm{G}$, a region-based data collection protocol for WSN. Its three main features are geographic routing, self-adaptation of the routing path based on energy awareness, and self-organization of the data collection and aggregation based on transaction cluster-heads, where a cluster corresponds to a geographical region. The results of data collection using $2 \mathrm{G}$ in both simulation and field experiments show that the novel feature of $2 \mathrm{G}$, namely its region-based self-organization using transaction clusterheads, leads to significant performance improvements compared to basic data collection using GEAR. In particular, this feature provides the benefits associated with clusters, while avoiding any overhead for cluster-head election and cluster maintenance. Our field experiments based on a real hardware test-bed validate the results of $2 \mathrm{G}$ in realistic conditions, which is promising for future developments.

The current version of the protocol is available on request. Present limitations are the absence of a flexible and accurate localization service, and the lack of accurate battery power measurements due to the API problems mentioned in the previous section. Future work should target these issues as they impact the usability of the routing protocol.

\section{ACKNOWLEDGMENTS}

This research was partially funded by special funding from the Japanese Society for Promotion of Science and the National Institute of Informatics.

\section{REFERENCES}

[1] K. Akkaya and M. F. Younis. A survey on routing protocols for wireless sensor networks. Ad Hoc Networks, 3(3):325-349, 2005.

[2] J. A. Aslam, Q. Li, and D. Rus. Three power-aware routing algorithms for sensor networks. Wireless Communications and Mobile Computing, 3(2):187-208, 2003.

[3] A. Awad, T. Frunzke, and F. Dressler. Adaptive distance estimation and localization in wsn using rssi measures. In $D S D$, pages 471-478. IEEE, 2007.

[4] L. Fang, W. Du, and P. Ning. A beacon-less location discovery scheme for wireless sensor networks. In INFOCOM, pages 161-171. IEEE, 2005.

[5] S. Hussain, A. W. Matin, and O. Islam. Genetic algorithm for energy efficient clusters in wireless sensor networks. In ITNG, pages 147-154. IEEE Computer Society, 2007.

[6] B. Karp and H. T. Kung. GPSR: greedy perimeter stateless routing for wireless networks. In MOBICOM, pages 243-254, 2000.

[7] B. Leong. New Techniques for Geographic Routing. PhD thesis, MIT CSAIL, Cambridge, MA 02139 USA, June 2006.

[8] P. Levis, N. Lee, M. Welsh, and D. E. Culler. Tossim: accurate and scalable simulation of entire tinyos applications. In I. F. Akyildiz, D. Estrin, D. E. Culler, and M. B. Srivastava, editors, SenSys, pages 126-137. ACM, 2003.

[9] Crossbow micaz platform, 2008. Accessed in October 2008.

[10] E. Platon and Y. Sei. Security software engineering in wireless sensor networks. Progress in Informatics, 5(1):1-19, 2008.

[11] K. Römer, C. Frank, P. J. Marrón, and C. Becker. Generic role assignment for wireless sensor networks. In Y. Berbers and M. Castro, editors, ACM SIGOPS European Workshop, page 2. ACM, 2004.

[12] A. Savvides, C.-C. Han, and M. B. Srivastava. Dynamic fine-grained localization in ad-hoc networks of sensors. In MOBICOM, pages 166-179, 2001.

[13] K. Seada, M. Zuniga, A. Helmy, and B. Krishnamachari. Energy-efficient forwarding strategies for geographic routing in lossy wireless sensor networks. In J. A. Stankovic, A. Arora, and R. Govindan, editors, SenSys, pages 108-121. ACM, 2004.

[14] Y. Xu, J. S. Heidemann, and D. Estrin. Geography-informed energy conservation for ad hoc routing. In MOBICOM, pages 70-84, 2001.

[15] Y. Yu, R. Govindan, and D. Estrin. Geographical and energy aware routing: A recursive data dissemination protocol for wireless sensor networks. Technical Report UCLA/CSD-TR-01-0023, UCLA Computer Science Department, May 2001. 